\title{
Observation of multilayer quantum Hall effect in the charge density wave material $\mathrm{CaCu}_{4} \mathrm{As}_{2}$
}

\author{
Souvik Sasmal $\odot,{ }^{1,}{ }^{*}$ Vikas Saini ${ }^{\circledR},{ }^{1}$ Sitaram Ramakrishnan $\odot,{ }^{2,3}$ Gourav Dwari $\odot,{ }^{1}$ Bishal Baran Maity $\odot,{ }^{1}$ Jin-Ke Bao $\odot,{ }^{2}$ \\ Rajib Mondal $\odot,{ }^{4}$ Vikram Tripathi, ${ }^{5}$ Sander van Smaalen $\odot,{ }^{2}$ Bahadur Singh $\odot,{ }^{1, \dagger}$ and A. Thamizhavel ${ }^{1, 末}$ \\ ${ }^{1}$ Department of Condensed Matter Physics and Materials Science, Tata Institute of Fundamental Research, Homi Bhabha Road, \\ Colaba, Mumbai 400005, India \\ ${ }^{2}$ Laboratory of Crystallography, University of Bayreuth, 95447 Bayreuth, Germany \\ ${ }^{3}$ Department of Quantum Matter, AdSM, Hiroshima University, Higashi-Hiroshima, 739-8530, Japan \\ ${ }^{4}$ UGC-DAE Consortium for Scientific Research, Kolkata Centre, Bidhannagar, Kolkata 700 106, India \\ ${ }^{5}$ Department of Theoretical Physics, Tata Institute of Fundamental Research, Homi Bhabha Road, Colaba, Mumbai 400005, India
}

(Received 14 July 2021; revised 1 December 2021; accepted 10 January 2022; published 7 February 2022)

\begin{abstract}
Low-dimensional layered materials manifest an inherent tendency towards the formation of symmetry reduced charge density wave (CDW) states with exotic properties. Here, we elucidate the anisotropic transport properties of $\mathrm{CaCu}_{4} \mathrm{As}_{2}$, which crystallizes in a rhombohedral lattice. Temperature-dependent single-crystal x-ray diffraction in conjunction with thermodynamic and transport measurements reveal that $\mathrm{CaCu}_{4} \mathrm{As}_{2}$ undergoes a structural or CDW transition below $51 \mathrm{~K}$. For $I \|$ [12 10$]$, angular-dependent Shubnikov-de Haas oscillations reveal a two-dimensional nature of the charge carriers contributing to the bulk transport in accord with our calculated Fermi surface. In the inverse Hall resistance versus $1 / B$ plot, quantized Hall plateaus are observed. $\mathrm{CaCu}_{4} \mathrm{As}_{2}$ thus provides a new platform to understand the coexistence of both the CDW and quantum Hall effect in materials.
\end{abstract}

DOI: 10.1103/PhysRevResearch.4.L012011

The quantum Hall effect (QHE) [1,2] and charge density wave (CDW) [3-5] are exotic physical properties that are generally observed in low-dimensional systems. The underlying mechanism of CDW lies in the one-dimensional (1D) chains [6-8] or two-dimensional (2D) layers [9-11] present in the crystal structure. In 2D materials, high magnetic fields at low temperatures lead to dissipationless edge states, producing the QHE. There have been ongoing efforts to realize quantized Hall resistance and CDW effects in three-dimensional (3D) solids. The charge carriers in a 3D solid are free to move in all three spatial directions which makes the quantization of Hall resistance difficult. Over the last few years, the obvious extension of $2 \mathrm{D}$ QHE to bulk materials has led to the discovery of QHE in bulk systems such as $\eta-\mathrm{Mo}_{4} \mathrm{O}_{11}$ [12], $n$-doped $\mathrm{Bi}_{2} \mathrm{Se}_{3}$ [13], EuMnBi 2 [14], and $\mathrm{BaMnSb}_{2}$ [15]. But the CDW state has not been reported in these materials. It was found that the QHE in Bechgaard salt is due to a spin density wave [16,17]. In $\mathrm{ZrTe}_{5}$, it was thought that the QHE is due to the CDW, although recent thermodynamic studies disregarded that mechanism $[18,19]$. Similarly, QHE in $\mathrm{HfTe}_{5}$ is due to strong electron interaction effects [20]. Here, we show that $\mathrm{CaCu}_{4} \mathrm{As}_{2}$ is a new material that shows both the CDW and QHE.

\footnotetext{
*sasmalsouvik6@gmail.com

†bahadur.singh@tifr.res.in

"thamizh@tifr.res.in
}

Published by the American Physical Society under the terms of the Creative Commons Attribution 4.0 International license. Further distribution of this work must maintain attribution to the author(s) and the published article's title, journal citation, and DOI.
$\mathrm{CaCu}_{4} \mathrm{As}_{2}$ adopts a rhombohedral lattice with space group $R \overline{3} m$ at room temperature. It possesses a layered crystal structure similar to $\mathrm{SrAg}_{4} \mathrm{As}_{2}$ [21], $\mathrm{EuAg}_{4} \mathrm{As}_{2}$ [22,23], and $\mathrm{SrCu}_{4} \mathrm{As}_{2}$ [24]. Below $51 \mathrm{~K}$, a CDW/structural transition is observed in our single-crystal $\mathrm{x}$-ray diffraction (SXRD) measurements that are well corroborated with the electrical transport and heat capacity studies. In the field range of 10-14 T, for $I \|[1 \overline{2} 10]$ and $B \|$ [0001], the Shubnikovde Haas $(\mathrm{SdH})$ oscillation provides an insight into the Fermi surface. The angular-dependent $\mathrm{SdH}$ oscillations imply a quasi-2D Fermi surface. In the inverse Hall resistance $\left(1 / R_{x y} Z\right)$ versus $1 / B$ plot, constant step-size Hall plateaus are observed, indicating QHE in the system. Here, $Z$ is the average number of $2 \mathrm{D}$ channels. Our experimental results reveal the presence of a large number of 2D conduction channels and a structural distortion as the source for the observed quantized Hall plateaus.

The unit cell of $\mathrm{CaCu}_{4} \mathrm{As}_{2}$ with lattice parameters $a=$ 4.1460(4) $\AA$ and $c=22.4517(6) \AA$ is shown in Fig. 1(a). It is composed of three formula units of $\mathrm{CaCu}_{4} \mathrm{As}_{2}$ with repeated septuple layer (SL) blocks [Cu1-Cu2-As-Ca-As-Cu2-Cu1]. The calculated bulk band structure of $\mathrm{CaCu}_{4} \mathrm{As}_{2}$ along the high-symmetry directions in the bulk Brillouin zone (BZ) is shown in Fig. 1(b). It exhibits a metallic character such that both the hole and electron bands cross the Fermi level. Importantly, the hole bands form corrugated cylindrical Fermi pockets, whereas the electron bands constitute open sheet Fermi pockets centered at the $F$ points [see the Fermi surface Fig. 1(c)]. The calculated Fermi surface of $\mathrm{CaCu}_{4} \mathrm{As}_{2}$ thus reveals its 2D-like nature, consistent with the $\mathrm{SdH}$ oscillation measurements presented below.

Single crystals of $\mathrm{CaCu}_{4} \mathrm{As}_{2}$ are grown by the flux method (see Supplemental Materials (SMs) Sec. S1 for details [25]). 

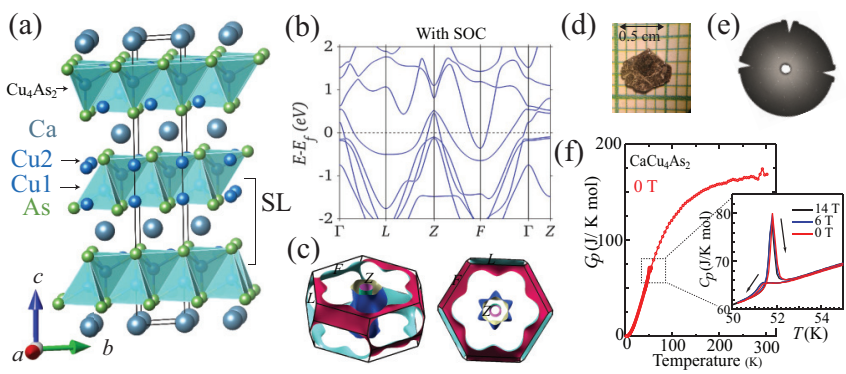

FIG. 1. (a) Crystal structure of $\mathrm{CaCu}_{4} \mathrm{As}_{2}$. (b) Calculated electronic structure of bulk $\mathrm{CaCu}_{4} \mathrm{As}_{2}$ with spin-orbit coupling (SOC). (c) The associated Fermi surface with three open Fermi pockets. (d,e) As-grown single crystal and its Laue diffraction pattern. (f) Heat capacity as a function of temperature. Inset shows the closeup region around the phase transition $52 \mathrm{~K}$.

The grown crystals are platelet-like with the flat plane corresponding to the (0001)-plane as shown in Fig. 1(d). The Laue diffraction pattern is shown in Fig. 1(e). The six-fold symmetry and well-defined spots confirm the good quality of the crystal. Figure 1(f) shows the heat capacity in the temperature range from $2-300 \mathrm{~K}$. The overall behavior of the heat capacity is similar to a typical nonmagnetic compound. At $52 \mathrm{~K}$, a broad peak is observed while cooling, and a sharp peak with a jump of about $15 \mathrm{~J} / \mathrm{K} \cdot \mathrm{mol}$ is observed while warming. The heat capacity measurements in the presence of the magnetic field up to $14 \mathrm{~T}$ do not have a significant effect on the observed anomaly. To confirm the CDW/structural distortion, we perform SXRD experiments. Details regarding crystal structure refinements in the periodic phase are given in Secs. S2 and S3 of the SMs [25]. Using CRYSALISPRO [26], the reciprocal layer of the $(h k 0)$ plane is reconstructed as shown in Figs. 2(a) and 2(b) at $80 \mathrm{~K}$ and $45 \mathrm{~K}$. Between the main Bragg reflections indicated by the indices, there are weak superlattice reflections at positions $\mathbf{q}^{\mathbf{1}}=[-0.0386(5), 0.0867(7), 0.2500(19)]$ and $\mathbf{q}^{2}=[0.0830(4),-0.0421(5), 0.2501(19)]$ in Fig. 2(b). Our analysis indicates that there are satellite reflections up to $m=8$, indicating its complexity (Sec. S3 of SMs [25]). Moreover, the structure is possibly twinned, suggesting a lowering of symmetry to either monoclinic or triclinic. Such a distortion of the lattice resulting in a change from high to low symmetry accompanied by superlattice reflections is not uncommon in CDW compounds and has occurred in recently investigated CDW compounds like $\mathrm{CuV}_{2} \mathrm{~S}_{4}$ [27] and $R_{2} \mathrm{Ir}_{3} \mathrm{Si}_{5}(R=\mathrm{Er}, \mathrm{Lu})$ [28,29].

The temperature variation of the electrical resistivity in the $a b$-plane $\left(\rho_{x x}\right)$ and along the $c$-axis $\left(\rho_{z z}\right)$ are shown in Figs. 2(c) to 2(f). The resistivity shows a metallic behavior and shows a huge anisotropy, thus signaling a quasi-2D character. At $51 \mathrm{~K}$, a sharp drop in the resistivity is observed in $\rho_{x x}$ and then it gradually decreases down to $2 \mathrm{~K}$. The sharp drop in the resistivity is first-order-like, as confirmed from the observed hysteresis [inset of Fig. 2(c)]. This drop in resistivity occurs due to a structural/CDW transition. Interestingly, such a drop in resistivity was observed in transition metal dichalcogenides (TMDCs) where the strong electron coupling in the electronic structure drives CDW and opens the partial gap in the Fermi surface [30-32]. On the other hand, $\rho_{z z}$ shows a small humplike feature at $51 \mathrm{~K}$ [Fig. 2(d)] and then decreases as the (a)
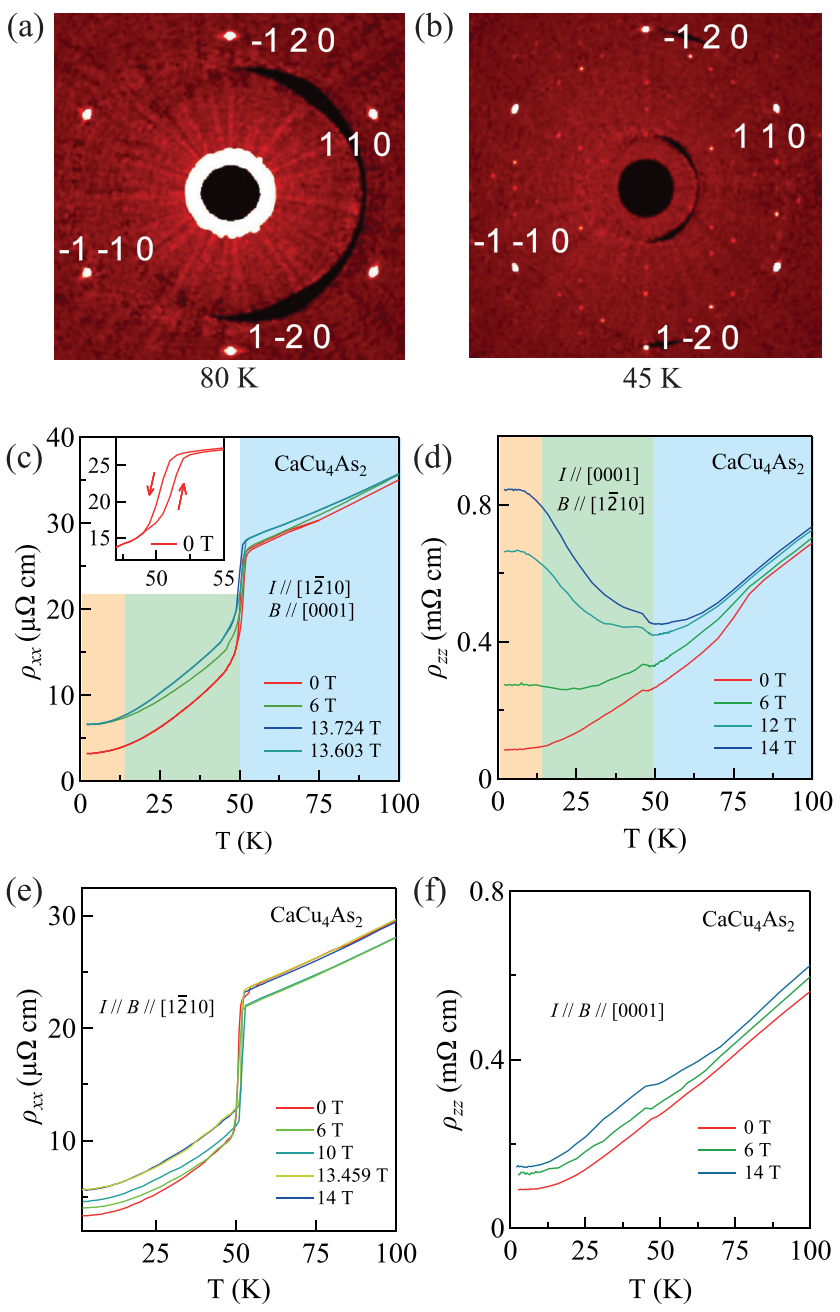

FIG. 2. SXRD data measured at (a) $80 \mathrm{~K}$ and (b) $45 \mathrm{~K}$. Superlattice reflections are observed at $45 \mathrm{~K}$ indicating the CDW. Black bands are unreactive gaps between modules of the Pilates $1 \mathrm{M} \mathrm{CdTe}$ detector. Panel (a) is slightly zoomed in as compared to panel (b) as the crystal-to-detector distance is greater in panel (b) to resolve the satellite reflections and possible twinning. (c)-(f) Electrical resistivity for different current and field directions. For $I \|[1 \overline{2} 10]$ and $B \|$ [0001], a sharp drop in resistivity at $51 \mathrm{~K}$ is observed. Inset in panel (c) shows a hysteresis during the heating and cooling cycle at $51 \mathrm{~K}$. (d) For $I \|$ [0001] and $B \|[1 \overline{2} 10]$, in the presence of an applied magnetic field, below $51 \mathrm{~K}$, the figure shows a slight increase in resistivity with decreasing temperatures, as is typically observed in CDW systems. (e) For $I\|B\|[1 \overline{2} 10]$, the resistivity behavior is almost similar to (c). (f) For $I\|B\|[0001]$ shows a very subtle anomaly at $51 \mathrm{~K}$ although the system is metallic.

temperature is lowered down to $2 \mathrm{~K}$. The small hump-like feature in the resistivity indicates structural distortion at the same temperature $51 \mathrm{~K}$. The applied transverse magnetic field (B \| [0001]) does not have any major effects for $I \|$ [12 10$]$, and the overall behavior of the electrical resistivity remains the same. On the other hand with transverse magnetic field along [1 $1 \overline{2} 10]$ the $\rho_{z z}$ reveals a drastic change and shows an upturn as temperature decreases [indicated by the $(\square)$ color], and it almost saturates below $15 \mathrm{~K}$ [indicated by the ( color]. The charge carriers, below the transition temperature, 

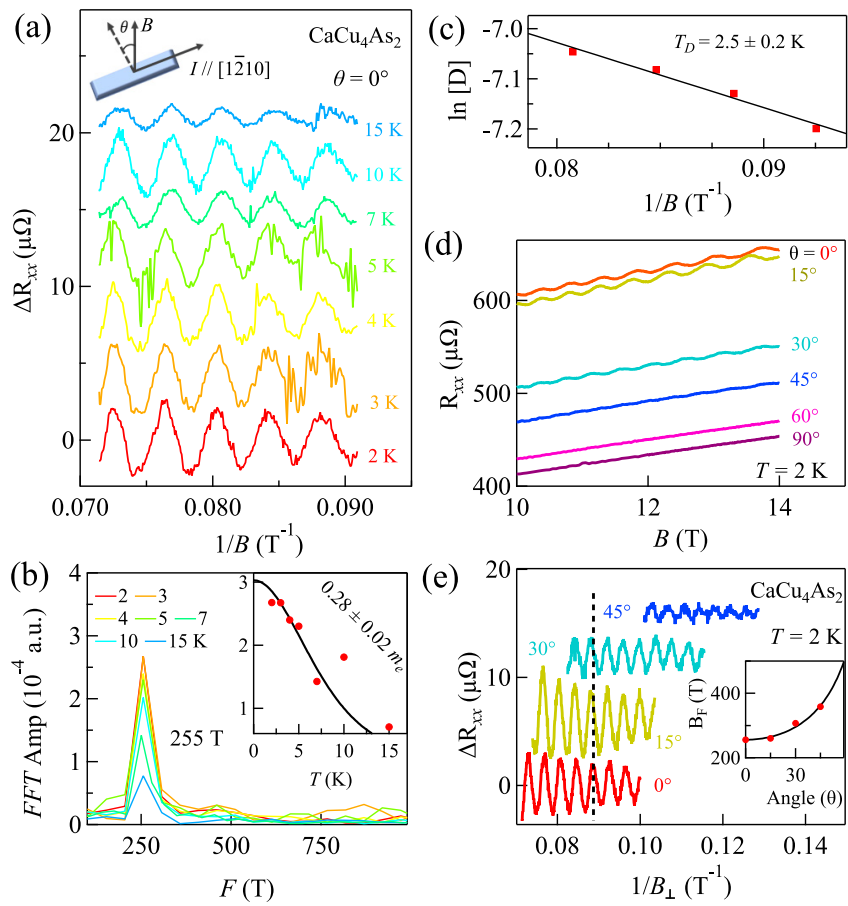

FIG. 3. (a) Background-subtracted resistance $\left(\Delta R_{x x}\right)$ at various temperature as a function of $1 / B$. (b) FFT spectrum at various temperature. Inset shows mass plot. (c) From the slope of linear fit of $\ln [D]$ versus $1 / B$ the $T_{D}$ is estimated. (d) Field-dependent longitudinal resistance $\left(R_{x x}\right)$ plotted at various angle $\left(0^{\circ} \leqslant \theta \leqslant 90^{\circ}\right)$. At high angles the oscillations vanish. (e) $\Delta R_{x x}$ is plotted as a function of $1 / B_{\perp}$. The dashed line shows that the maxima position in $\Delta R_{x x}$ is almost same for different angles. Inset shows that FFT frequency follows the cosine behavior, which is shown with the solid line.

are more confined in $a b$ plane and a larger scattering occurs along the $c$-direction in the presence of magnetic field so that the estimated anisotropy $\left(\rho_{z z} / \rho_{x x}\right)$ is 117 at $2 \mathrm{~K}$ and $14 \mathrm{~T}$. This field-induced effect suggests the $c$-direction is poorly conducting in comparison to the $a b$-plane. Figures $2(\mathrm{e})$ and 2(f) show the longitudinal magnetoresistance where the current and magnetic field are parallel to each other. For $I \|$ $B \|$ [12̄10], the magnetic field does not have any effect on the overall electrical resistivity. While for $I\|B\|$ [0001], the field-induced metal-insulator-like behavior is absent and the resistivity displaysed a metallic behavior. The resistivity $\rho_{x x}$ and $\rho_{z z}$ in transverse and longitudinal field configurations are shown in Fig. S9 [25].

For $I \|[1 \overline{2} 10]$ and $B \|[0001]$ the magnetoresistance shows $\mathrm{SdH}$ oscillation in the field range of 10-14 $\mathrm{T}$. Figure 3(a) illustrates the background-subtracted $\mathrm{SdH}$ oscillations $\left(\Delta R_{x x}\right)$, for $I \|[1 \overline{2} 10]$ and $B \|$ [0001], measured at different temperatures in the field range of 10-14 T. The temperature dependence of the fast Fourier transformation (FFT) of the SdH oscillation is shown in Fig. 3(b). The same FFT frequency has been observed in another sample (Fig. S11 in SMs [25]). Notably, to observe oscillation from other pockets, a much lower temperature and higher field is necessary. The $\mathrm{SdH}$ oscillation can be described by the Lifshitz-Kosevich (LK) expression [33]. Figure 3(b) shows the temperature dependence of the FFT amplitude. According to the LK expression, the temperature dependence of the oscillation amplitude follows the thermal damping factor $\lambda / \sinh (\lambda)$, where $\lambda=2 \pi^{2}\left(k_{B} T m^{*} / \hbar e B\right)$. Here $T$ is temperature, $k_{B}$ is the Boltzmann constant, and $m^{*}$ is the effective mass. From the temperature dependence of the oscillation amplitude, we find the effective mass $m^{*}$ as $0.28 \pm 0.02 m_{e}$ [Inset of Fig. 3(b)]. The Dingle temperature $\left(T_{\mathrm{D}}\right)$ is obtained from the slope of linear fit of $\ln [D]$ versus $1 / B$. Here, $D$ is defined as $(A B \sin h[\lambda(T, B)])$ where $A$ is the oscillation amplitude. At $2 \mathrm{~K}$, from the linear slope, we find $T_{\mathrm{D}}$ to be around $2.5 \pm 0.2 \mathrm{~K}$ [Fig. 3(c)]. In high pure crystalline samples, the less impurity scattering collision broadening of the electronic state at the Fermi level may cause such a low $T_{D}$. From the $\mathrm{SdH}$ oscillation, considering the spin-degeneracy 2 , we estimated $2 \mathrm{D}$ carrier density $n_{\mathrm{SdH}}^{2 \mathrm{D}}=\left(2 e B_{F}\right) / h \sim 12.4 \times 10^{16} \mathrm{~m}^{-2}$, Fermi velocity $v_{F}=$ $\left(\hbar k_{F} / m^{*}\right) \sim 3.64 \times 10^{5} \mathrm{~m} / \mathrm{sec}$, and the quantum mobility $\mu_{\mathrm{SdH}}=(h e) /\left(4 \pi^{2} K_{B} T_{D} m^{*}\right) \sim 0.31 \mathrm{~m}^{2} \mathrm{~V}^{-1} \mathrm{~s}^{-1}$, where $B_{F}$ is the oscillation frequency $(255 \mathrm{~T}), h$ is the Planck's constant, and $e$ is the electron charge. The obtained value of $v_{F}$ is in close agreement with the estimated values in the $a b$-plane of $\mathrm{KCu}_{4} \mathrm{As}_{2}$ and $\mathrm{SrCu}_{4} \mathrm{As}_{2}$, where a similar type of corrugated cylindrical Fermi pockets reveal the 2D character [24].

The angular dependence of the $\mathrm{SdH}$ oscillation is shown in Fig. 3(d). The oscillation amplitude decreases with increasing angle between magnetic field and normal to the surface $(\theta)$ and it starts vanishing at an angle $\theta \geqslant 30^{\circ}$. In Fig. 3(e), the plot of $\Delta R_{x x}$ measured at various angles as a function of $1 / B_{\perp}=1 / B \cos \theta$ shows all maxima and minima falling on the same position of $1 / B_{\perp}$. FFT frequencies for all angles plotted in the inset of Fig. 3(e) follows the $1 / B_{\perp}$ or $1 / B \cos \theta$ behavior, suggesting the $\mathrm{SdH}$ oscillation originated from the 2D character of the charge carriers. The disappearance of the $\mathrm{SdH}$ oscillation at lower angles gives additional support to the quasi-2D nature of the Fermi surface. Based on our band structure, we calculate the angular dependence of quantum oscillations of various Fermi pockets (see Fig. S12 of SMs [25]). We find that the experimentally observed frequency corresponds to the central cylindrical hole pocket located along the $\Gamma-Z$ line, indicating the 2D nature of observed Fermi surface. This open cylindrical Fermi pocket is different from Refs. [34-36], where the quasi-2D charge transport originated from the elongated ellipsoidal 3D Fermi pockets. A Landau level fan diagram is shown in Fig. S13.

Figure 4(a) shows the antisymmetrized Hall resistivity plot $\rho_{x y}\left(\left[\rho_{x y}^{\mathrm{raw}}(+B)-\rho_{x y}^{\mathrm{raw}}(-B)\right] / 2\right)$ measured at various temperatures for $I \|[1 \overline{2} 10]$ and $B \|[0001]$. From the linear slope, the Hall carrier density $\left(n_{\text {Hall }}\right)$ and the Hall mobility $\left(\mu_{\text {Hall }}\right)$ are estimated as $4.8 \times 10^{26} \mathrm{~m}^{-3}$ and $0.5 \mathrm{~m}^{2} \mathrm{~V}^{-1} \mathrm{~s}^{-1}$, respectively. The inset of Fig. 4(a) shows a change in the carrier density at $51 \mathrm{~K}$ since the formation of the structural transition affects the linear slope and hence the carrier density. $\Delta R_{x x}$ and $1 /\left(R_{x y} Z\right)$ are plotted as a function of $1 / B$ [Fig. 4(b)], which shows that the Hall plateau arises when minima occurs in longitudinal resistance. Notably, QHE is generally observed in $2 \mathrm{D}$ systems. In $2 \mathrm{D}$ electron gas, the carrier localization is due to impurities or defects. With the increase in magnetic field the Landau orbital size of the charge carriers become smaller, and thus leading to carrier localization in these impurities or defects. Here, the observation of the plateaus in Hall resistivity can be thought of as a multilayer QHE in 

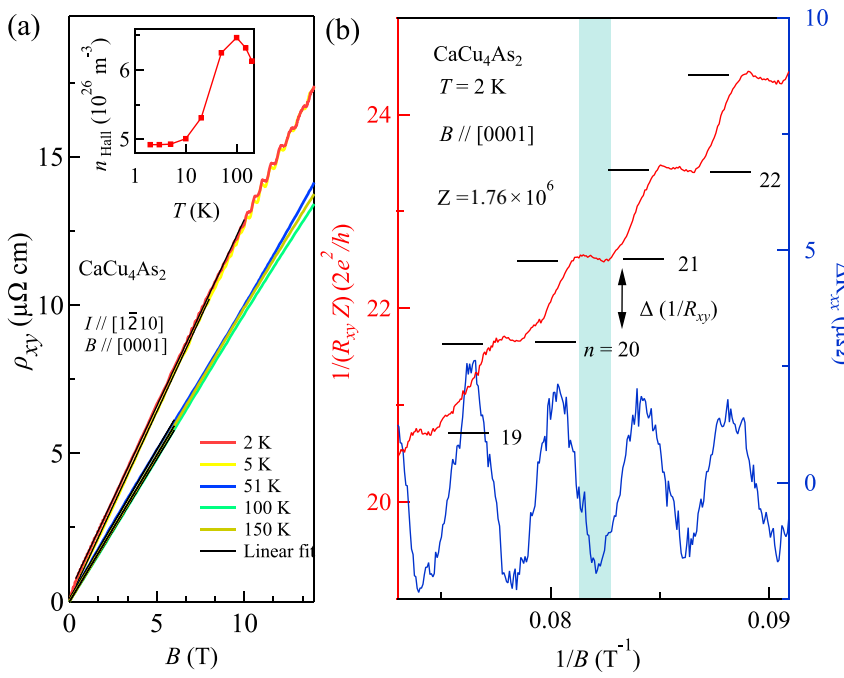

FIG. 4. (a) Hall resistivity $\left(\rho_{x y}\right)$ measured at different temperatures; inset: a significant change in carrier concentration is observed at CDW transition $51 \mathrm{~K}$. (b) Minima position of $\Delta R_{x x}$ is found when $1 /\left(R_{x y} Z\right)$ plateau arises [indicated by $(\square)$ color] which suggests approximately $90^{\circ}$ phase difference between background-subtracted longitudinal resistance and Hall. Landau quantized plateaus are indicated as $n$.

$\mathrm{CaCu}_{4} \mathrm{As}_{2}$. Furthermore, the conditions such as $\omega_{c} \tau$ should be greater than 1 and $\hbar \omega_{c} \gg k_{\mathrm{B}} T$ has to be satisfied to observe QHE. From our estimated quantum parameters, we find that $\omega_{c} \tau=\left(\frac{e B}{m^{*}}\right)\left(\frac{\hbar}{2 \pi k_{B} T_{D}}\right) \simeq 4.3$ and $\hbar \omega_{c}=\hbar\left(\frac{e B}{m^{*}}\right)=5.7 \mathrm{meV}$, for a magnetic field of $14 \mathrm{~T}$ and $k_{\mathrm{B}} T=0.17 \mathrm{meV}$ for $T=2 \mathrm{~K}$, showing that both the conditions are satisfied. This suggests that the observed plateaus in the Hall resistivity are indeed the signatures of $\mathrm{QHE}$ in $\mathrm{CaCu}_{4} \mathrm{As}_{2}$. Additionally, charge carrier separation $\left(1 / \sqrt{n_{\mathrm{SdH}}} \sim 4 \mathrm{~nm}\right)$ is comparable to magnetic length scale $(\sqrt{\hbar / e B} \sim 6.8 \mathrm{~nm})$, implying we are close to the quantum limit.

For the multilayer quantum Hall effect, the Hall resistance can be expressed as $R_{x y}=\frac{1}{n Z}\left(h / 2 e^{2}\right)$, where $n\left(=B_{F} / B\right)$ is the Landau filling factor and $Z$ is the average number of $2 \mathrm{D}$ spin-degenerate channels contributing to the conduction. $Z$ can be estimated from the step size of the two successive Hall plateaus $\Delta\left(\frac{1}{R_{x y}}\right)=\left(\frac{1}{n}-\frac{1}{n+1}\right) \frac{1}{R_{x y}}=137 \Omega^{-1}$. Since $\Delta\left(\frac{1}{R_{x y}}\right)=$ $Z \frac{2 e^{2}}{h}$, from the obtained values $\Delta\left(\frac{1}{R_{x y}}\right)$ we estimate the average number of conduction channel $Z$ values as $1.76 \times 10^{6}$. Using the obtained $Z$ value, we plot $\left(\frac{1}{R_{x y} Z}\right)\left(\frac{2 e^{2}}{h}\right)$ versus $\frac{1}{B}$ in Fig. 4(b) and obtain a step size of about 0.92, which is close to 1 . This indicates that each $2 \mathrm{D}$ conduction channel contributes about $2 e^{2} / h$ which infers that there are $Z$ parallel $2 \mathrm{D}$ conduction channels in the system. The sample thickness (t) is $410 \mu \mathrm{m}$, and thus the number of $2 \mathrm{D}$ conduction channels per unit thickness $Z / t$ is $4.3 \times 10^{9} \mathrm{~m}^{-1}$. The calculated bulk carrier density is $n_{\mathrm{Bulk}}=n_{\mathrm{SdH}}^{2 \mathrm{D}} \times Z / t=5.3 \times 10^{26} \mathrm{~m}^{-3}$. Also, the carrier density obtained from Hall slope $n_{\text {Hall }}$ is $4.8 \times 10^{26} \mathrm{~m}^{-3}$. Two independently calculated quantity $n_{\mathrm{Bulk}}$ and $n_{\text {Hall }}$ are nearly equal, suggesting $2 \mathrm{D}$ conduction channels are the reason for such plateaus in the Hall data. Again, the $\mu_{\mathrm{SdH}}$ and $\mu_{\mathrm{Hall}}$ are also almost the same. Similar quantized Hall plateaus are observed in Hall conductivity (Fig. S14
[25]) and another sample (Fig. S15 [25]). This observed 2D nature of the carriers is consistent with our calculated Fermi surface, which is also found to be 2D. The complex structure of $\mathrm{CaCu}_{4} \mathrm{As}_{2}$ helps to develop the metal bonding in the form of extensive network of $\left[\mathrm{Cu}_{4} \mathrm{As}_{2}\right]$ - slabs (see the discussion in SMs Sec. S1 [25]). The $\left[\mathrm{Cu}_{4} \mathrm{As}_{2}\right]$ slab width is approximately $3.4 \AA$. Although, it is not confirmed how the conduction channels are distributed, the experimental result shows the conduction channel width $(t / Z)$ is approximately $2.3 \AA$, which is nearly equal to the $\left[\mathrm{Cu}_{4} \mathrm{As}_{2}\right]$ slab width. The isostructural $\mathrm{SrAg}_{4} \mathrm{As}_{2}$ has also metal-metal bonding in the form of the $2 \mathrm{D}$ network $\left[\mathrm{Ag}_{4} \mathrm{As}_{2}\right]$ slab and the Sr states are mostly empty above the Fermi level [37].

The formation of the CDW, in general, can be attributed to the Fermi surface nesting that opens a partial gap in the Fermi surface. Such a gap opening decreases the number of charge carriers at the Fermi level and thus increases the electrical resistivity. When the current is passed in the basal plane $(a b)$, the resistivity drops sharply. On the other hand, when the current is carried along the $c$-axis, there is a minuscule hump followed by a decrease. This anomalous behavior in the electrical resistivity gives evidence for the CDW formation in a way similar to the $2 H$ phase of TMDCs where the sudden drop in the electrical resistivity is seen and attributed to the CDW formation [30]. Rice and Scott [38] showed theoretically that the unusual behavior of the electrical resistivity occurs due to the partial gap opening in the Fermi surface owing to the presence of saddle-points at the Fermi energy. In contrast, Shen et al. [31,32], presented another explanation of the CDW using the notion of the Fermi patch from careful angle-resolved photoelectron spectroscopy (ARPES) studies. The systems with CDW ordering have more states across the Fermi patches than the ones without CDW order. Across the transition, the CDW gap opens over the Fermi patches but not at the Fermi surfaces, thus resulting in a metallic state. $\mathrm{CaCu}_{4} \mathrm{As}_{2}$ has a band structure with saddle-points as well as substantial dispersionless bands along $\Gamma-Z$ direction close to the Fermi level. Thus, both the aforementioned mechanisms may play a role in the formation of CDW. ARPES along with a comprehensive analysis of the crystal structure below $51 \mathrm{~K}$ is necessary to understand its mechanism.

Considering the observed Hall resistivity plateaus, the CDW causes a periodic carrier modulation, which, in turn, generates disorder in the system and results in the localized states. These localized states that appear between the Landau levels pin the chemical potential over large magnetic fields, leading to the plateaus in Hall resistance. The presence of quantized Hall steps in a 3D system might be ascribed to the system's large number of 2D conduction channels. From the analysis of the Hall resistivity data, we find that each conduction channel contributes about $\left(2 e^{2} / h\right)$ to the Hall resistivity. It was reported in $\mathrm{Bi}_{2} \mathrm{Se}_{3}$ that each two quintuple layer (QL) contributes $\sim\left(2 e^{2} / h\right)$ to the Hall resistivity [13]. Similarly, a recent study showed that in $\mathrm{MnBi}_{2} \mathrm{Te}_{4}$, which adopts weakly coupled SLs, these SLs contributes to the quantized Hall resistivity $[39,40]$. For $\mathrm{CaCu}_{4} \mathrm{As}_{2},\left[\mathrm{Cu}_{4} \mathrm{As}_{2}\right]$ slabs play a crucial role in $2 \mathrm{D}$ conduction.

In conclusion, the angular-dependent $\mathrm{SdH}$ oscillation studies demonstrate that the Fermi surface is quasi-2D consistent with our first-principles calculations. We observe a 
structural/CDW transition at $51 \mathrm{~K}$ and $\mathrm{QHE}$ in this bulk material. Based on the Hall data analysis, we find the quantized step size, revealing an integer QHE in $\mathrm{CaCu}_{4} \mathrm{As}_{2}$. The observation of the CDW and QHE in bulk $\mathrm{CaCu}_{4} \mathrm{As}_{2}$ will open new research avenues in experimental and theoretical physics.

We thank A. Schaller, S. Kotla and L. Noohinejad for their assistance in collecting SXRD data at Beamline P24. We acknowledge DESY (Hamburg, Germany), a member of the Helmholtz Association HGF, for the provision of experimental facilities. Parts of this research were carried out at PETRA III, using beamline P24. Beamtime was allocated for proposal I-20200805. We acknowledge the Department of Atomic Energy (DAE) of the Government of India for financial support. J.-K.B. acknowledges Alexander von Humboldt Foundation for the financial support in Germany.
[1] K. V. Klitzing, G. Dorda, and M. Pepper, New Method for HighAccuracy Determination of the Fine-Structure Constant Based on Quantized Hall Resistance, Phys. Rev. Lett. 45, 494 (1980).

[2] K. S. Novoselov, Z. Jiang, Y. Zhang, S. V. Morozov, H. L. Stormer, U. Zeitler, J. C. Maan, G. S. Boebinger, P. Kim, and A. K. Geim, Room-temperature quantum hall effect in graphene, Science 315, 1379 (2007).

[3] R. E. Peierls, Quantum Theory of Solids (Clarendon, New York, 1996).

[4] H. Fröhlich, On the theory of superconductivity: The onedimensional case, Proc. R. Soc. Lond. Ser. A, Math. Phys. Sci. 223, 296 (1954).

[5] G. Gruner, Density Waves in Solids (CRC Press, Boca Raton, FL, 2018).

[6] W. Shi, B. J. Wieder, H. L. Meyerheim, Y. Sun, Y. Zhang, Y. Li, L. Shen, Y. Qi, L. Yang, J. Jena, P. Werner, K. Koepernik, S. Parkin, Y. Chen, C. Felser, B. A. Bernevig, and Z. Wang, A charge-density-wave topological semimetal, Nat. Phys. 17, 381 (2021).

[7] H. Yi, Z. Huang, W. Shi, L. Min, R. Wu, C. M. Polley, R. Zhang, Y.-F. Zhao, L.-J. Zhou, J. Adell, X. Gui, W. Xie, M. H. W. Chan, Z. Mao, Z. Wang, W. Wu, and C.-Z. Chang, Surface charge induced Dirac band splitting in a charge density wave material $\left(\mathrm{TaSe}_{4}\right)_{2}$ I, Phys. Rev. Res. 3, 013271 (2021).

[8] F. Galli, S. Ramakrishnan, T. Taniguchi, G. J. Nieuwenhuys, J. A. Mydosh, S. Geupel, J. Lüdecke, and S. van Smaalen, Charge-Density-Wave Transitions in the Local-Moment Magnet $\mathrm{Er}_{5} \mathrm{Ir}_{4} \mathrm{Si}_{10}$, Phys. Rev. Lett. 85, 158 (2000).

[9] T. Ritschel, J. Trinckauf, K. Koepernik, B. Büchner, M. V. Zimmermann, H. Berger, Y. I. Joe, P. Abbamonte, and J. Geck, Orbital textures and charge density waves in transition metal dichalcogenides, Nat. Phys. 11, 328 (2015).

[10] C. Brun, Z.-Z. Wang, P. Monceau, and S. Brazovskii, Surface Charge Density Wave Phase Transition in $\mathrm{NbSe}_{3}$, Phys. Rev. Lett. 104, 256403 (2010).

[11] A. V. Melechko, J. Braun, H. H. Weitering, and E. W. Plummer, Two-Dimensional Phase Transition Mediated by Extrinsic Defects, Phys. Rev. Lett. 83, 999 (1999).

[12] S. Hill, S. Uji, M. Takashita, C. Terakura, T. Terashima, H. Aoki, J. S. Brooks, Z. Fisk, and J. Sarrao, Bulk quantum Hall effect in $\eta-\mathrm{Mo}_{4} \mathrm{O}_{11}$, Phys. Rev. B 58, 10778 (1998).

[13] H. Cao, J. Tian, I. Miotkowski, T. Shen, J. Hu, S. Qiao, and Y. P. Chen, Quantized Hall Effect and Shubnikov-de Haas Oscillations in Highly Doped $\mathrm{Bi}_{2} \mathrm{Se}_{3}$ : Evidence for Layered Transport of Bulk Carriers, Phys. Rev. Lett. 108, 216803 (2012).
[14] H. Masuda, H. Sakai, M. Tokunaga, Y. Yamasaki, A. Miyake, J. Shiogai, S. Nakamura, S. Awaji, A. Tsukazaki, H. Nakao, Y. Murakami, T.-h. Arima, Y. Tokura, and S. Ishiwata, Quantum Hall effect in a bulk antiferromagnet $\mathrm{EuMnBi}_{2}$ with magnetically confined two-dimensional Dirac fermions, Sci. Adv. 2, e1501117 (2016).

[15] J. Y. Liu, J. Yu, J. L. Ning, H. M. Yi, L. Miao, L. J. Min, Y. F. Zhao, W. Ning, K. A. Lopez, Y. L. Zhu, T. Pillsbury, Y. B. Zhang, Y. Wang, J. Hu, H. B. Cao, B. C. Chakoumakos, F. Balakirev, F. Weickert, M. Jaime, Y. Lai et al., Spin-valley locking and bulk quantum Hall effect in a noncentrosymmetric Dirac semimetal BaMnSb ${ }_{2}$, Nat. Commun. 12, 4062 (2021).

[16] S. T. Hannahs, J. S. Brooks, W. Kang, L. Y. Chiang, and P. M. Chaikin, Quantum Hall Effect in a Bulk Crystal, Phys. Rev. Lett. 63, 1988 (1989).

[17] L. Balents and M. P. A. Fisher, Chiral Surface States in the Bulk Quantum Hall Effect, Phys. Rev. Lett. 76, 2782 (1996).

[18] F. Tang, Y. Ren, P. Wang, R. Zhong, J. Schneeloch, S. A. Yang, K. Yang, P. A. Lee, G. Gu, Z. Qiao, and L. Zhang, Threedimensional quantum Hall effect and metal-insulator transition in $\mathrm{ZrTe}_{5}$, Nature (London) 569, 537 (2019).

[19] S. Galeski, T. Ehmcke, R. Wawrzyńczak, P. M. Lozano, K. Cho, A. Sharma, S. Das, F. Küster, P. Sessi, M. Brando, R. Küchler, A. Markou, M. König, P. Swekis, C. Felser, Y. Sassa, Q. Li, G. Gu, M. V. Zimmermann, O. Ivashko et al., Origin of the quasi-quantized Hall effect in $\mathrm{ZrTe}_{5}$, Nat. Commun. 12, 3197 (2021).

[20] S. Galeski, X. Zhao, R. Wawrzyńczak, T. Meng, T. Förster, P. M. Lozano, S. Honnali, N. Lamba, T. Ehmcke, A. Markou, Q. Li., G. Gu, W. Zhu, J. Wosnitza, C. Felser, G. F. Chen, and J. Gooth, Unconventional Hall response in the quantum limit of $\mathrm{HfTe}_{5}$, Nat. Commun. 11, 5926 (2020).

[21] B. Shen, E. Emmanouilidou, X. Deng, A. McCollam, J. Xing, G. Kotliar, A. I. Coldea, and N. Ni, Significant change in the electronic behavior associated with structural distortions in monocrystalline $\mathrm{SrAg}_{4} \mathrm{As}_{2}$, Phys. Rev. B 98, 235130 (2018).

[22] B. Shen, C. Hu, H. Cao, X. Gui, E. Emmanouilidou, W. Xie, and N. Ni, Structural distortion and incommensurate noncollinear magnetism in $\mathrm{EuAg}_{4} \mathrm{As}_{2}$, Phys. Rev. Mater. 4, 064419 (2020).

[23] S. L. Bud'ko, L. Xiang, C. Hu, B. Shen, N. Ni, and P. C. Canfield, Pressure tuning of structural and magnetic transitions in $\mathrm{EuAg}_{4} \mathrm{As}_{2}$, Phys. Rev. B 101, 195112 (2020).

[24] V. G. Hadjiev, B. Lv, and C. W. Chu, Electronic band structure of $\mathrm{SrCu}_{4} \mathrm{As}_{2}$ and $\mathrm{KCu}_{4} \mathrm{As}_{2}$ : Metals with diversely doped $\mathrm{CuAs}$ layers, Phys. Rev. B 84, 073105 (2011).

[25] See Supplemental Material at http://link.aps.org/supplemental/ 10.1103/PhysRevResearch.4.L012011 for the method, powder 
$\mathrm{x}$-ray diffraction, crystal structure, single-crystal x-ray diffraction, Shubnikov-de Haas oscillation, and quantum Hall effect in an other sample.

[26] CRYSALIS pro version 171.40.53, Rigaku Oxford Diffraction.

[27] S. Ramakrishnan, A. Schönleber, C. B. Hübschle, C. Eisele, A. M. Schaller, T. Rekis, N. H. A. Bui, F. Feulner, S. van Smaalen, B. Bag, S. Ramakrishnan, M. Tolkiehn, and C. Paulmann, Charge density wave and lock-in transitions of $\mathrm{CuV}_{2} \mathrm{~S}_{4}$, Phys. Rev. B 99, 195140 (2019).

[28] S. Ramakrishnan, A. Schönleber, T. Rekis, N. van Well, L. Noohinejad, S. van Smaalen, M. Tolkiehn, C. Paulmann, B. Bag, A. Thamizhavel, D. Pal, and S. Ramakrishnan, Unusual charge density wave transition and absence of magnetic ordering in $\mathrm{Er}_{2} \mathrm{Ir}_{3} \mathrm{Si}_{5}$, Phys. Rev. B 101, 060101(R) (2020).

[29] S. Ramakrishnan, A. Schönleber, J.-K. Bao, T. Rekis, S. R. Kotla, A. M. Schaller, S. van Smaalen, L. Noohinejad, M. Tolkiehn, C. Paulmann, N. S. Sangeetha, D. Pal, A. Thamizhavel, and S. Ramakrishnan, Modulated crystal structure of the atypical charge density wave state of single-crystal $\mathrm{Lu}_{2} \mathrm{Ir}_{3} \mathrm{Si}_{5}$, Phys. Rev. B 104, 054116 (2021).

[30] B. Ruzicka, L. Degiorgi, H. Berger, R. Gaál, and L. Forró, Charge Dynamics of $2 \mathrm{H}-\mathrm{TaSe}_{2}$ along the Less-Conducting c-Axis, Phys. Rev. Lett. 86, 4136 (2001).

[31] D. W. Shen, B. P. Xie, J. F. Zhao, L. X. Yang, L. Fang, J. Shi, R. H. He, D. H. Lu, H. H. Wen, and D. L. Feng, Novel Mechanism of a Charge Density Wave in a Transition Metal Dichalcogenide, Phys. Rev. Lett. 99, 216404 (2007).

[32] D. W. Shen, Y. Zhang, L. X. Yang, J. Wei, H. W. Ou, J. K. Dong, B. P. Xie, C. He, J. F. Zhao, B. Zhou, M. Arita, K. Shimada,
H. Namatame, M. Taniguchi, J. Shi, and D. L. Feng, Primary Role of the Barely Occupied States in the Charge Density Wave Formation of $\mathrm{NbSe}_{2}$, Phys. Rev. Lett. 101, 226406 (2008).

[33] D. Shoenberg, Magnetic Oscillations in Metals (Cambridge University Press, Cambridge, England, 2009).

[34] A. K. Okazaki, S. Wiedmann, S. Pezzini, M. L. Peres, P. H. O. Rappl, and E. Abramof, Shubnikov-de Haas oscillations in topological crystalline insulator $\operatorname{SnTe}(111)$ epitaxial films, Phys. Rev. B 98, 195136 (2018).

[35] B. A. Piot, W. Desrat, D. K. Maude, M. Orlita, M. Potemski, G. Martinez, and Y. S. Hor, Hole Fermi surface in $\mathrm{Bi}_{2} \mathrm{Se}_{3}$ probed by quantum oscillations, Phys. Rev. B 93, 155206 (2016).

[36] S. Sasmal, V. Saini, N. Bruyant, R. Mondal, R. Kulkarni, B. Singh, V. Tripathi, and A. Thamizhavel, Weak antilocalization and Shubnikov-de Haas oscillations in single crystal $\mathrm{CaCuSb}$, Phys. Rev. B 104, 205135 (2021).

[37] S. S. Stoyko, M. Khatun, C. Scott Mullen, and A. Mar, Ternary $\mathrm{CaCu}_{4} \mathrm{P}_{2}$-type pnictides $\mathrm{AAg}_{4} \mathrm{Pn}_{2}(\mathrm{~A}=\mathrm{Sr}, \mathrm{Eu} ; \mathrm{Pn}=\mathrm{As}, \mathrm{Sb})$, J. Solid State Chem. 192, 325 (2012).

[38] T. M. Rice and G. K. Scott, New Mechanism for a ChargeDensity-Wave Instability, Phys. Rev. Lett. 35, 120 (1975).

[39] Y. Deng, Y. Yu, M. Z. Shi, Z. Guo, Z. Xu, J. Wang, X. H. Chen, and Y. Zhang, Quantum anomalous Hall effect in intrinsic magnetic topological insulator $\mathrm{MnBi}_{2} \mathrm{Te}_{4}$, Science 367, 895 (2020).

[40] C. Liu, Y. Wang, H. Li, Y. Wu, Y. Li, J. Li, K. He, Y. Xu, J. Zhang, and Y. Wang, Robust axion insulator and Chern insulator phases in a two-dimensional antiferromagnetic topological insulator, Nat. Mater. 19, 522 (2020). 